\title{
BRD4 is a Histone Acetyltransferase that Evicts Nucleosomes from Chromatin
}

\author{
Ballachanda N. Devaiah ${ }^{1}$, Chanelle Case-Borden ${ }^{1}$, Anne Gegonne ${ }^{1}$, Chih Hao Hsu², \\ Qingrong Chen ${ }^{2}$, Daoud Meerzaman ${ }^{2}$, Anup Dey ${ }^{3}$, Keiko Ozato $^{3}$, and Dinah S. Singer ${ }^{1}$ \\ ${ }^{1}$ Experimental Immunology Branch, $\mathrm{NCI}, \mathrm{NIH}$, Bethesda, Maryland, USA \\ ${ }^{2}$ Center for Biomedical Informatics and Information Technology, $\mathrm{NCl}, \mathrm{NIH}$, Bethesda, Maryland, \\ USA \\ ${ }^{3}$ Laboratory of Molecular Growth Regulation, NICHD, NIH, Bethesda, Maryland, USA
}

\begin{abstract}
Bromodomain protein 4 (BRD4) is a chromatin-binding protein implicated in cancer and autoimmune diseases that functions as a scaffold for transcription factors at promoters and superenhancers. Whereas chromatin de-compaction and transcriptional activation of target genes are associated with BRD4 binding, the mechanism(s) involved are unknown. We report that BRD4 is a novel histone acetyltransferase (HAT) that acetylates histones $\mathrm{H} 3$ and $\mathrm{H} 4$ with a pattern distinct from other HAT's. Both mouse and human BRD4 demonstrate intrinsic HAT activity. Importantly, BRD4 acetylates H3K122, a residue critical for nucleosome stability, resulting in nucleosome eviction and chromatin de-compaction. Nucleosome clearance by BRD4 occurs genome-wide, including at its targets $M Y C, F O S$ and $A U R K B$ (Aurora B kinase), resulting in increased transcription. Since BRD4 regulates transcription, these findings lead to a model where BRD4 actively links chromatin structure and transcription: It mediates chromatin de-compaction by acetylating and evicting nucleosomes of target genes, thereby activating their transcription.
\end{abstract}

\section{Keywords}

BRD4; Histone acetyltransferase; Histone H3 acetylation; Nucleosome eviction; Chromatin decompaction

\footnotetext{
Users may view, print, copy, and download text and data-mine the content in such documents, for the purposes of academic research, subject always to the full Conditions of use: http://www.nature.com/authors/editorial_policies/license.html\#terms

Corresponding author: Dinah S. Singer, Experimental Immunology Branch, CCR, National Cancer Institute, NIH, Bethesda, MD 20892, Phone: 301-496-9097, FAX: 301-480-0977, dinah.singer@nih.gov.

ACCESSION CODES

MNase-seq and RNA-seq data was deposited in the Gene Expression Omnibus database under the omnibus accession code GSE71577 AUTHOR CONTRIBUTIONS

B.N.D. performed the experimental work with C.C. doing microscopy analysis and BRD4(fl/fl) MEF analysis. A.G. generated and isolated BRD4 KO thymocytes and did FACS analysis. B.N.D and D.S. designed experiments, analyzed results and wrote the manuscript. A.D. and K.O. generated the BRD4fl/fl mice. C.H, Q.C and D.M did whole-genome sequence analysis and statistics.

COMPETING FINANCIAL INTERESTS

The authors declare no competing financial interests.
} 


\section{INTRODUCTION}

BRD4 plays an important role in cellular functions such as transcription, replication, epigenetic regulation and DNA repair ${ }^{1}$. It was originally identified as a chromatin-binding scaffold that recruits transcription factors and chromatin modifiers ${ }^{2,3}$. It also functions as a mitotic bookmark of genes transcriptionally reactivated in early $\mathrm{G} 1^{4,5}$, including $M Y C, F O S$ and $A U R K B^{4,6-8}$. Further studies revealed that BRD4 binds to promoters and nucleates super-enhancers ${ }^{9}$, suggesting an active role in gene regulation. Indeed, BRD4 not only passively scaffolds transcription factors such as $\mathrm{PTEFb}$ and $\mathrm{NF \kappa B}$, but also actively regulates transcription elongation ${ }^{10-12}$.

Transcriptional activation depends on acetylation of nucleosomal histones and chromatin decompaction around targeted genes, making them accessible to transcription factors ${ }^{13,14}$. Acetylation neutralizes the positive charge of lysine residues on nucleosomal histones, weakening their interactions with DNA and adjacent nucleosomes ${ }^{15}$. Specific histone acetylation marks also serve as docking sites for chromatin adapters and modifiers described as 'readers' ${ }^{16,17}$. BRD4 is one such reader preferentially recruited to histones acetylated at $\mathrm{H} 3 \mathrm{~K} 14, \mathrm{H} 4 \mathrm{~K} 5$, or H4K12 10,18 . While the role of acetylation of the amino-terminal tail lysines positioned on the surface of nucleosomes has been extensively analyzed, less is known about the effect of acetylating lysines located within histone carboxy-terminal globular domains. Modifications of lysines within the globular domain, which lie on the lateral surface of the histone octamer in direct contact with DNA, can destabilize the nucleosome and affect overall chromatin architecture ${ }^{19}$. Acetylation of histone H3 Lys122 (H3K122), positioned on the dyad axis of the nucleosome where the DNA-histone bond is strongest, causes histone eviction and nucleosome instability ${ }^{20}$. H3K122ac on chromatinized templates is associated with increased transcription rates in vitro ${ }^{20}$. In vivo, $\mathrm{H} 3 \mathrm{~K} 122 \mathrm{ac}$ is concentrated at active enhancers and promoters, correlating with transcription; absence of H3K122ac is associated with transcriptional silencing. Currently, p300 and CBP are the only histone acetyltransferases (HAT's) known to acetylate H3K122 ${ }^{20}$. Blocking BRD4 interaction with histones using the I-BET inhibitor reduces histone acetylation levels at promoter regions ${ }^{21}$. Additionally, depletion of BRD4 leads to selective hypoacetylation of histone $\mathrm{H} 3$ and $\mathrm{H} 4{ }^{22}$.

All known HAT's dissociate from the chromatin during mitosis ${ }^{23}$, leading to the question of how mitotic chromosomes are re-acetylated for chromatin de-compaction and transcriptional reactivation. Intriguingly, BRD4 overexpression resulted in chromatin de-compaction and accelerated transcriptional reactivation post-mitosis ${ }^{24}$. However, BRD4 does not interact with, or recruit, any of the known HAT's, including p300, GCN5, PCAF, CBP or Tip607,25.

The above observations led us to investigate whether BRD4 has HAT activity and plays an active role in mediating chromatin de-compaction. We report that BRD4 has intrinsic HAT activity that acetylates the tail lysines of nucleosomal histone $\mathrm{H} 3$ and H4. Importantly, BRD4 also acetylates the H3K122 residue in the globular core of the histone octamer, resulting in histone eviction, nucleosome clearance and chromatin de-compaction with a correlated increase in nuclear volume and transcription. Genome-wide, H3K122ac colocalizes with BRD4. BRD4-mediated nucleosome clearance is dependent on its HAT 
activity and targets genomic loci where BRD4 is known to bind. Furthermore, BRD4 depletion, and consequent decrease in histone acetylation, correlates with aberrant thymic development in vivo. These studies identify an active role for BRD4 in regulating chromatin structure, leading to a model in which BRD4's roles in chromatin de-compaction and transcription are integrated.

\section{RESULTS}

\section{BRD4 has intrinsic acetyltransferase activity}

Since many bromodomain proteins (e.g. CBP, TAF1) are known HAT's, we speculated that BRD4 may also have HAT activity. Indeed, recombinant murine BRD4 efficiently acetylated histones H3 and H4 (Fig. 1a; Supplementary Fig. 1a); BRD4 also auto-acetylated (Fig. 1a, Supplementary Fig. 1b). BRD4 HAT activity was consistently detected independent of source or method of purification (Fig. 1b). Importantly, the BRD4 HAT activity is intrinsic to it: In an in-gel HAT assay with murine and human BRD4, acetylated $\mathrm{H} 4$ peptides were only associated with BRD4 (Fig. 1c).

We next examined BRD4's substrate specificity. BRD4 acetylated all four core histones, although it preferentially acetylated $\mathrm{H} 3$ and $\mathrm{H} 4-$ as well as histone $\mathrm{H} 1$ (Fig. 1d, Supplementary Fig. 1c,d). In contrast, BRD4 did not acetylate any of its non-histone partners that we examined (Supplementary Table 1). Therefore, the primary targets of BRD4 acetyltransferase activity are histones, defining BRD4 as a histone acetyltransferase. BRD4 also efficiently acetylated mononucleosomes assembled in vitro on 5S rDNA, with a preference for nucleosomal H3 (Fig. 1e). BRD4 acetylation of nucleosomal histones contrasts with that of p300, which preferentially acetylates H4 (Fig. 1e). Furthermore, metaanalysis of ChIP-Seq data demonstrated that inhibition of BRD4 binding to chromatin by either one of two small molecule BRD4 inhibitors, iBET or JQ1, resulted in decreased histone acetylation (Supplementary Fig. 2a). BRD4 overexpression in HeLa cells resulted in a global increase in acetylation levels of $\mathrm{H} 3$ and $\mathrm{H} 4$ (average of 2.2 and 2.3 times in magnitude, respectively) (Fig. 1f). Indeed, BRD4 overexpression resulted in increased H3 acetylation in multiple cell lines of both human and murine origin, demonstrating that BRD4 acetylates histones in a wide range of cell types (Supplementary Fig. 2b). Thus, BRD4 is an acetyltransferase that acetylates nucleosomal histones both in vitro and in vivo.

\section{BRD4 HAT activity maps to predicted acetyl CoA binding sites}

We next mapped the BRD4 domains responsible for its HAT activity. Two regions of murine BRD4 (BRD4) matched the highly conserved motif A, a known acetyl CoA recognition and binding sequence, $(\mathrm{R} / \mathrm{Q}) \mathrm{XXGX}(\mathrm{G} / \mathrm{A})^{26}$ : one site (amino acids $\left.175-180\right)$ lies in the Nterminus adjacent to bromodomain 1; the other (amino acids 1097-1102) is in the Cterminus. Point mutations in either site abrogated BRD4 HAT activity (Fig. 2a). Thus, HAT activity of BRD4 maps to sites homologous to AcCoA binding sites. BRD4 has no significant sequence similarity to catalytic domains of known HATs. However, deletion of a 40 amino acid segment (aa 1157-1197) in the C-terminus4 abolished BRD4 HAT activity ( $\triangle 40 \mathrm{AA})$. In contrast, deletion of a 60 amino acid segment (aa1340-1400; $\triangle 60 \mathrm{AA})$ spanning the interaction sites of proteins such as PTEFb and HPV E2 did not affect BRD4 
HAT activity. Combinations of these mutations abrogated HAT activity (Fig. 2a; lower panel). BRD4 mutants deleted of either or both bromodomains retained HAT activity (Fig. 2b). Therefore, BRD4 HAT activity depends on a 40 aa region in the C-terminus, which likely contains the catalytic domain and on two sites homologous to AcCoA binding sites, but not on the bromodomains. Introduction of homologous mutations into human BRD4 ( $\triangle$ NT-CoA Bs and $\triangle 40 \mathrm{AA}$ ) similarly abrogated its HAT activity (see ahead to Fig. $4 \mathrm{f}$ ).

BRD4 HAT mutants retained their ability to bind histones (Fig. 2c). BRD4 bound unacetylated histones, albeit with low affinity relative to acetylated histones ${ }^{18}$; this binding was significantly enhanced in the presence of AcCoA (Supplementary Fig. 2c). Further, JQ1, an acetyl lysine mimic that binds BRD4 bromodomains and inhibits binding to histones ${ }^{27}$, significantly reduced acetylation (Supplementary Fig. 3a). At high concentrations, JQ1 also blocked the HAT activity of the BRD4 mutant that lacks both bromodomains, suggesting that JQ1 can directly inhibit BRD4 catalytic activity (Supplementary Fig. 3a)

\section{BRD4 HAT differs from known histone acetyltransferases}

We next determined which lysine residues in the N-terminal tails of $\mathrm{H} 3$ and $\mathrm{H} 4$ are acetylated by BRD4, because differential acetylation can lead to distinct functional consequences ${ }^{16}$. BRD4 efficiently acetylated H3K4, H3K9, H3K18 and H3K27, all marks associated with open chromatin and active transcription (Fig. 3a). Like p300, BRD4 acetylated $\mathrm{H} 4$ at K5, K8, K12 and K16. However, whereas p300 acetylated H4 K5, K8 and K12 residues with comparable efficiency, BRD4 preferentially acetylated K8>K5>K12>K16 (Fig. 3a). Interestingly, H4K8 is strongly acetylated by BRD4, but not by TAF1, demonstrating that BRD4 and TAF1 HAT activities are distinct. Most importantly, BRD4 did not acetylate either lysine H3K14 or H3K56 as detected by immunoblotting with specific antibodies (Fig. 3a). This is of particular significance since all other known H3 acetyltransferases acetylate H3K14 15,16,28. This pattern of $\mathrm{H} 3$ acetylation distinguishes BRD4 from other HATs. While BRD4 is capable of acetylating most $\mathrm{H} 3$ tail lysines and all $\mathrm{H} 4$ tail lysines, it does so with different kinetics, demonstrating its selectivity in vitro (Supplementary Figs. 3b and 3c).

Although H3K14 is not detectably acetylated by BRD4, acetylated H3K14 is one of the marks that stimulates BRD4 binding to $\mathrm{H} 3{ }^{18}$. Indeed, BRD4 acetylation of $\mathrm{H} 3$ peptides was more efficient when the peptides were pre-acetylated at K14 compared to either unacetylated or H3K9-acetylated peptide controls (Supplementary Fig. 3d). Thus, although H3K14 is not a substrate for acetylation by BRD4, the acetylated H3K14 residue is a docking site for BRD4, positioning it for subsequent acetylation of other lysine residues.

The patterns of BRD4 acetylation of $\mathrm{H} 3$ and $\mathrm{H} 4$ lysine residues observed in vitro were also observed in vivo. Expression of exogenous BRD4 resulted in increased acetylation of H3K4, H3K9, H3K18 and H3K27, relative to the control cells (Fig. 3b). Increased acetylation of H4K5, H4K8, H4K12 and H4K16 was also observed (Fig. 3c). Importantly, BRD4 overexpression cells did not affect H3K14 levels (Fig. 3b). Since all previously known HATs acetylate H3K14, the absence of increased H3K14ac upon BRD4 overexpression indicates that the increased acetylation at other histone lysine residues is a direct effect of BRD4 HAT 
activity. These patterns of acetylation define a BRD4 HAT 'fingerprint'. Furthermore, BRD4 overexpression led to a significant increase in nuclear H3K9ac indicating that BRD4 acetylates chromatin-bound H3 (Fig. 3d). Thus, BRD4 acetylates histones in vitro and in vivo.

BRD4 HAT activity has a distinct inhibition profile relative to other known HATs. The broad spectrum HAT inhibitors anacardic acid and garcinol inhibited BRD4 acetylation of both H3 and H4, but curcumin did not (Fig. 3e), thereby distinguishing it from other HATs. BRD4 HAT activity increased with increasing $\mathrm{pH}$, showing the highest activity between $\mathrm{pH} 8.0$ and pH 9.0 (Supplementary Fig. 3e). This is consistent with previous findings that histone acetylation correlates with intracellular $\mathrm{pH}^{29}$.

\section{BRD4 acetylates H3K122 in the globular domain of histone $\mathrm{H3}$}

The increase in nuclear volume accompanying in vivo overexpression of BRD4 (Fig. 3d, lower panel) suggested that BRD4 mediates chromatin de-compaction and nucleosome eviction. Nucleosome eviction depends on acetylation of H3K122 in the globular domain in the histone octamer (Fig. 4a) ${ }^{20}$. Strikingly, BRD4 acetylated H3K122 in vitro (Fig. 4b). Mass spectrometric analysis confirmed that BRD4 acetylates the H3K122 residue (Supplementary Fig. 4a). In addition, overexpression of BRD4 markedly increased H3K122ac levels in vivo (Fig. 4c, Supplementary Fig. 4b). Increased H3K122 acetylation depended on BRD4 HAT activity: none of the HAT-defective mutants increased H3K122ac levels, whereas both the $\Delta 60$ AA mutant, which retains HAT activity, and wild type BRD4 did (Supplementary Fig. 4b). H3K14ac levels, which are not targeted by BRD4, remained unchanged.

Conversely, depletion of BRD4 in HeLa cells by siRNA led to a 51\% decrease in H3K122ac levels; decreases in the more labile H3K18ac and H4K5ac were even greater (90\% and 73\% respectively) (Fig. 4d). The decrease in H3K122ac was particularly striking since it is a very stable mark whose turn-over is normally undetectable in cells ${ }^{30}$, suggesting that BRD4 maintains H3K122ac levels. We speculate that the residual H3K122ac observed in BRD4depleted cells is maintained by p300/CBP. Notably, H3K14ac and H3K56ac levels remained unchanged.

BRD4 acetylation of H3K122 was independent of p300 and CBP, the only HATs known previously to acetylate H3K122, as shown in two different ways. First, siRNA depletion of p300 and CBP reduced, but did not eliminated, total H3K122ac levels. The residual H3K122ac likely reflects endogenous BRD4 HAT activity. Strikingly, exogenously expressed BRD4 restored H3K122ac levels in cells depleted of p300 and CBP to control levels; the BRD4 HAT mutant did not (Fig. 4e). Second, cells expressing exogenous WT or mutant BRD4 were treated with curcumin, a potent inhibitor of p300 and CBP but not BRD4 HAT activity (Supplementary Fig. 4c and Fig. 4f). Curcumin treatment reduced H3K122ac levels in the control and BRD4 HAT mutant transfected cells, reflecting the loss of p300 and CBP activity. However, exogenous WT BRD4 largely restored H3K122ac levels (>70\%) (Fig. 4f). In both sets of experiments, H3K56ac, an acetylation mark created exclusively by p300 and CBP, was completely eliminated, demonstrating the complete loss 
of p300 and CBP activity. Therefore, we conclude that BRD4 acetylates H3K122 independent of p300 and CBP.

To further document the direct role of BRD4 in acetylating histone $\mathrm{H} 3$, cells transfected with exogenous WT BRD4 or vector control were treated with increasing amounts of the BRD4 bromodomain inhibitor, JQ1 (Fig. 4g). JQ1 caused a dramatic decrease in H3K122ac and H3K18ac levels both in control cells expressing only endogenous BRD4 and in cells expressing exogenous BRD4 (Fig. 4g, Supplementary Fig. 4d). Of note, although cells depleted of p300 and CBP had reduced H3K122ac, JQ1 inhibition of BRD4 binding to chromatin reduced H3K122ac levels even further (Supplementary Fig. 5a). H3K14ac, an acetylation mark not mediated by BRD4, was unaffected in JQ1 treated cells. Thus, BRD4 contributes to acetylation of $\mathrm{H} 3 \mathrm{~K} 122 \mathrm{ac}$ and $\mathrm{H} 3 \mathrm{~K} 18$ through its binding to chromatin. Supporting these results, in vivo H3K122ac levels correlate with BRD4 genome-wide, as evidenced by meta-analysis of ChIP-seq data where $>93 \%$ of BRD4 bound sites co-localize with H3K122ac peaks (Fig. 4h). Furthermore, H3K122ac levels peak at sites of maximal BRD4 binding (Fig. 4i); neither the intensity nor pattern of this co-localization is observed either with other transcription factors or with p300 and CBP (Supplementary Fig. 5b). Taken together, these results demonstrate that BRD4 acetylates H3K122 in vivo. BRD4 is the only HAT, other than p300 and CBP, shown to acetylate this critical residue.

\section{BRD4 mediates histone eviction and nucleosome clearance}

The findings that BRD4 acetylates $\mathrm{H} 3 \mathrm{~K} 122$, as well as the tails of both $\mathrm{H} 3$ and $\mathrm{H} 4$, and that its overexpression leads to increased nuclear volume, led us to speculate that BRD4 HAT activity contributes to nucleosomal disassembly. Indeed, BRD4 mediated the dissociation of mononucleosomes and the release of free DNA in the presence of acetyl CoA, accompanied by the acetylation of nucleosomal H3 at H3K9 and H3K122 (Fig. 5a). The addition of JQ1 to the reaction prevented mononucleosomal disruption. TAF1, another double bromodomain HAT that acetylates histone tail lysines, did not acetylate H3K122 or release DNA from the mononucleosomes (Fig. 5a). BRD4 mutants lacking HAT activity were unable to mediate mononucleosome disruption or acetylate mononucleosomal H3K9 and H3K122 (Fig. 5b). The $\triangle 60$ AA BRD4 mutant which retained HAT activity also retained the ability to disrupt the mononucleosomes (Fig. 5b). Together, these results demonstrate that BRD4 acetylation of mononucleosomal H3K122 mediates nucleosome disassembly.

We next tested if BRD4 similarly evicts nucleosomes from native chromatin by assessing BRD4-mediated changes in micrococcal nuclease (MNase) sensitivity which increases with decreased nucleosome occupancy. Chromatin incubated in vitro with increasing amounts of BRD4 was increasingly sensitive to MNase digestion; increased MNase digestion of chromatin depended on the presence of AcCoA and was inhibited by JQ1 (Fig. 5c). Thus, BRD4 disrupts nucleosomes from native chromatin in vitro, creating stretches of open DNA. BRD4 also mediated nucleosome clearance from chromatin in vivo. Exogenous expression of wild type, but not HAT mutant, BRD4 in U2OS cells resulted in a marked increase in MNase digestion of chromatin, relative to the control (Fig. 5d). Accordingly, higher levels of $\mathrm{H} 3 \mathrm{~K} 122 \mathrm{ac}$ and $\mathrm{H} 3 \mathrm{~K} 9 \mathrm{ac}$ were observed in these cells relative to the cells expressing the 
BRD4 HAT mutant or controls (Fig. 5d). We conclude that BRD4 HAT activity mediates clearance of nucleosomes from chromatin in vivo.

\section{BRD4 HAT modulates nucleosomal occupancy genome-wide}

We next asked whether BRD4 regulated nucleosome occupancy genome wide by MNaseseq analysis. Strikingly, wild type, but not mutant, BRD4 expression significantly reduced nucleosome occupancy downstream of transcription start sites (Fig. 6a and Supplementary Fig. 5c). Accordingly, BRD4 substantially increased RNA expression levels genome-wide as a function of reduced nucleosome occupancy (Fig. 6b). Thus, BRD4 HAT activity contributes to the reduced nucleosome occupancy associated with transcriptionally active genes.

BRD4 binds to and plays a critical role in regulating expression of proto-oncogenic M/G1 cell cycle regulators, e.g. $M Y C, F O S$ and $A U R K B^{2,7,8}$. Indeed, BRD4, but not the HAT mutant, decreased nucleosome occupancy around the transcription start sites (TSS) of $M Y C$, $F O S$ and $A U R K B$ genes (Fig. 6c). The sites of BRD4 HAT-mediated nucleosome clearance correspond to those sites where BRD4 has been shown to bind ${ }^{7}$. Thus, nucleosome clearance by BRD4 can be highly localized. In contrast, nucleosome occupancy around AURKA gene $^{8}$, which is BRD4-independent, was only marginally affected by BRD4 (Fig. 6c). Concomitant with BRD4-effected reductions in nucleosome occupancy, RNA levels of $M Y C, F O S$ and $A U R K B$ were significantly increased (Fig. 6d). We conclude that BRD4 mediates the nucleosome clearance that precedes transcriptional activation at its target genes.

We next determined whether BRD4 binding around the transcription start sites (TSS) of MYC, AURKB and AURKA resulted in nucleosomal H3K122 acetylation in vivo (Fig. 6e). H3K122ac levels, but not H3K14ac levels, increased in parallel with WT BRD4 occupancy at the $M Y C$ and $A U R K B$ loci but not at AURKA loci. Strikingly, JQ1 treatment reversed the increase in H3K122ac seen with WT BRD4 expression, with no effect either on H3K14ac levels or at the AURKA locus. In contrast, the BRD4 HAT mutant, which bound as efficiently as WT BRD4 to the target loci, did not cause an increase in H3K122ac (Fig. 6e). Consistent with the observed increases in RNA levels following BRD4 overexpression, increases in Pol II occupancy were observed around the $M Y C$ and $A U R K B$, but not $A U R K A$, loci (Supplementary Fig. 6). We conclude that BRD4 directly acetylates nucleosomal histones, leading to the clearance of nucleosomes from its target loci and providing access to the transcription machinery.

The effects of BRD4 on H3K122 acetylation and chromatin de-compaction can also be visualized directly. Introduction of wild type hBRD4, but not the hBRD4 mutant lacking HAT activity (Mt BRD4) in U2OS cells, resulted in a dramatic increase in H3K122 acetylation, as determined by immunofluorescence and increased nuclear volume, an indicator of chromatin de-compaction (Fig. 7a, b). Consistent with the inability of BRD4 to acetylate H3K14, H3K14ac levels were unchanged. 


\section{BRD4 deletion reduces H3K122ac levels in the thymus}

We next examined the effect of BRD4 deletion in vivo on H3K122ac levels and murine thymic development. Immature thymocytes which express neither cell surface molecules, CD4 or CD8 (double negative, DN), transition into thymocytes expressing both CD4 and CD8 (double positive, DP) and finally into cells expressing either CD4 or CD8. The transition from DN to DP is accompanied by extensive proliferation and differentiation, where BRD4 may be predicted to play a role.

Conditional deletion of BRD4 in the thymus (BRD4 KO) was achieved by expressing Cre from the lck promoter which is active only in early DN thymocytes. Thymocytes from the BRD4 KO mice had no detectable BRD4 protein (Fig. 7c). Relative to the thymus from either control or BRD4 heterozygotes, the BRD4 $4^{--}$thymus had a dramatically different profile of CD4 and CD8 expression, as assessed by flow cytometry (Fig. 7c). BRD4 deletion resulted in an increase in the proportion of immature, DN thymocytes (from 3\% to 15\%) and a corresponding decrease in the more mature DP cells (from 85\% to 60\%). Thus, BRD4 is required for normal development of thymocytes beyond the DN stage.

H3K122ac levels were markedly, albeit modestly (33\%), reduced in the thymus of BRD4 deficient mice. This modest reduction is consistent with both the long half-life of H3K122ac and its continued acetylation by $\mathrm{p} 300$ and $\mathrm{CBP}^{30}$. Accordingly, levels of the more labile $\mathrm{H} 3 \mathrm{~K} 18$ and $\mathrm{H} 4 \mathrm{~K} 5$ acetylations were dramatically reduced (85\% and $61 \%$ respectively). Levels of H3K14ac, which has a short half-life, remained unchanged consistent with the reductions in H2K122ac, H3K18ac and H4K5ac reflecting the loss of BRD4 (Fig. 7c).

To further document the effect of BRD4 deletion on acetylation of H3K122 in vivo, mouse embryonic fibroblasts (MEFs) were generated from heterozygous BRD4 $\mathrm{fl}^{/-}$mice. The ability of exogenous BRD4 to restore H3K122ac following Cre deletion of endogenous BRD4 was examined. Deletion of BRD4 by Cre resulted in cell death; cell viability could be rescued by exogenous BRD4. Exogenous wild type BRD4 completely restored H3K122ac levels, whereas the HAT mutant was unable to do so (49\% of parental levels) (Fig. 7d); the levels of exogenous BRD4 proteins were comparable to the original endogenous levels (Supplementary Fig. 7). The levels of H3K122ac observed in MEFs rescued with the HAT mutant were comparable to those observed when BRD4 was silenced (Fig. 4d), suggesting that in both cases the residual H3K122ac derives from p300 and CBP. Therefore, BRD4 HAT activity contributes to in vivo H3K122ac levels.

\section{DISCUSSION}

BRD4, originally identified associated with mitotic chromosomes and implicated in cell cycle control ${ }^{18}$, has key roles as a mitotic bookmark and a passive scaffold for transcription factors (reviewed in ref. ${ }^{31}$ ). However, BRD4 is also an active participant in transcription through its intrinsic kinase activity that phosphorylates Ser2 of the Pol II CTD ${ }^{11,32}$. The present study extends its role by demonstrating that BRD4 has an intrinsic HAT activity distinct from other known acetyltransferases. BRD4 acetylates lysines on the tails of $\mathrm{H} 3$ and $\mathrm{H} 4$ and, importantly, on H3K122, a lysine residue positioned in the globular domain of the histone octamer where the histone-DNA interaction is strongest. BRD4 co-localizes with 
H3K122ac genome-wide and its acetylation of $\mathrm{H} 3$ leads to nucleosomal disassembly and nuclear expansion, consistent with chromatin de-compaction. BRD4 preferentially reduces nucleosome occupancy around genes it is known to bind to and regulate such as $M Y C, F O S$ and $A U R K B$. Furthermore, conditional deletion of BRD4 in the thymus results in reduced $\mathrm{H} 3 \mathrm{~K} 122 \mathrm{ac}$ levels and perturbations in thymic development. Thus, BRD4 is an active remodeler of chromatin, as well as an active transcriptional regulator.

Nucleosomal disassembly and chromatin de-compaction mediated by BRD4 HAT activity occur preferentially at its target loci and at sites where BRD4 binds and where H3K122ac is enriched $^{7,20,33}$. While BRD4 de-compacted chromatin at its known targets such as $M Y C$, $F O S$ and $A U R K B$ genes, it did not markedly affect nucleosome occupancy around the $A U R K A$ gene which it does not regulate ${ }^{8}$. A previously reported $>95 \%$ correlation between BRD4 occupancy and DNaseI hypersensitivity (DHS) sites genome-wide ${ }^{34}$ is consistent with our conclusion that BRD4 clears nucleosomes at its target gene loci.

The finding that BRD4 HAT activity de-compacts chromatin resolves an apparent paradox in the literature. It was reported that a truncated isoform of BRD4 insulates chromatin from decompaction $^{3}$. We suggest that this isoform, which lacks the HAT catalytic domain, is a competitive inhibitor of full-length BRD4 which has HAT activity. Accordingly, overexpression of the truncated BRD4 isoform, containing only the $\mathrm{N}$-terminal bromodomains, resulted in the aggregation of chromatin into highly compact regions ${ }^{35}$. However, depletion of BRD4 resulted in increased MNase sensitivity, leading the authors to conclude that BRD4 is necessary for higher-order chromatin structure ${ }^{35}$. We speculate that this effect was not due to BRD4-mediated compaction but rather to chromothripsis induced either by premature chromosome condensation or inability to de-condense ${ }^{36}$. In other words, BRD4 overexpression and depletion both lead to altered chromatin structures, but mediated by different causes.

BRD4 is also a critical regulator of transcription, both by recruiting PTEFb to promoters and by phosphorylating Ser2 of the Pol II CTD through its intrinsic kinase activity, a modification necessary for transcriptional elongation ${ }^{11}$. Our discovery that BRD4 has intrinsic HAT activity, that regulates chromatin remodeling, leads to the conclusion that BRD4 actively links chromatin remodeling and transcription and to the following model (Fig. 8). We speculate that BRD4 contributes to remodeling of chromatin: 1) through its binding to $\mathrm{H} 3 \mathrm{~K} 14 \mathrm{ac}$ at promoters or enhancers and 2) by acetylation of both tail lysines and H3K122 in the globular domain of adjacent histone octamers. This leads to nucleosome eviction around the transcription start sites of target genes. The reduced nucleosomal occupancy allows access to the transcriptional machinery and recruitment of transcription factors, including PTEFb, resulting in activation of transcription. After transcription initiation, BRD4 triggers pause release by its phosphorylation of Pol II CTD 32,37 (Fig. 8). Future experiments will focus on defining the molecular mechanisms that regulate BRD4 HAT activity.

In conclusion, we demonstrate that BRD4actively regulates chromatin structure through its HAT activity and speculate that BRD4 links chromatin structure - through its acetylation and eviction of nucleosomal histones - with transcription through its phosphorylation of the Pol 
II CTD. Additionally, the discovery of BRD4 HAT activity should enable the development of novel inhibitors that specifically target its HAT activity for the treatment of cancers and autoimmune diseases.

\section{ONLINE METHODS}

Original uncropped images of gels, autoradiographs and blots used in this study can be found in Supplementary Data Set 1.

\section{Cell Lines and Culture}

HeLa, U2OS, DLD1, MCF-7, NIH3T3 and Mouse kidney cells (kind gift of Dr. Thomas Reid, NCI, NIH) were grown in DMEM media with $10 \% \mathrm{FBS}$ at $37{ }^{\circ} \mathrm{C}$ and $7.5 \% \mathrm{CO}$. Cell lines were tested for mycoplasma contamination and authenticated by spectral karyotyping or arrayCGH. Drosophila SF9 cells were grown at $27^{\circ} \mathrm{C}$ in TNM-FH insect medium (BD Biosciences Pharmingen).

\section{Plasmid constructs}

Murine Flag-His-BRD4 WT and $\triangle \mathrm{B} 1, \Delta \mathrm{B} 2$ and $\triangle \mathrm{B} 1 \mathrm{~B} 2$ mutants were described previously ${ }^{11}$. 6X His-BRD4-Flag $\triangle 40 \mathrm{aa}$ and $\triangle 60$ aa mutants were generated as drop-out mutants; $\triangle \mathrm{NT}$ CoA Bs, $\Delta \mathrm{CT}$ CoA Bs, $\Delta$ NT-CT CoA Bs BRD4 were generated as point mutants using primers with site specific mutations. $\Delta 40$ aa-NT CoA Bs, $\Delta 40$ aa-CT CoA Bs and $\triangle 40$ aa-NT-CT CoA Bs BRD4 mutants were generated using a combination of drop-out and site specific mutations with the BRD4 WT construct as template. WT BRD4 and mutants generated in the pACHLTc insect vector were moved into the mammalian pCDNA3.1 vector by excising the BRD4 sequences with BamH1 and SmaI and subcloning them into pCDNA3.1 using BamH1 and EcoRV. The GST-tagged human BRD4 in pGEX-6P-1 was from the Addgene repository (Addgene plasmid 14447). Human Flag tagged BRD4 WT in the pCMV2 mammalian vector was a gift from E. Verdin (Gladstone Institute of Virology, University of California, San Francisco). The hBRD4 $\Delta 40$ aa-NT CoA Bs double mutant was generated using the WT hBRD4 in the pCMV2 vector as a PCR template. The Flag-dTAF1 construct used to make recombinant TAF1 in Sf9 cells was described previously ${ }^{38}$.

\section{Transient transfections}

Transient transfections into mammalian cells were done using Lipofectamine (Invitrogen). Cells were treated with $5 \mathrm{mM}$ Sodium butyrate during transfection to inhibit HDAC activity and harvested $18 \mathrm{~h}$ post transfection. Whole cell extracts were analyzed by immunoblotting using specific antibodies as indicated in the figure legends. BRD4 knockdown was done by Lipofectamine-mediated transfection of 80nM siRNA (ON-TARGETplus smartpool human BRD4 or siGENOME Non-targeting siRNA pool, Dharmacon) into HeLa cells. Whole cell extracts were made at 24,48 and $72 \mathrm{~h}$ after siRNA transfection and used for immunoblotting. CBP and p300 knockdowns were done by transfecting $10 \mathrm{nM}, 25 \mathrm{nM}$ or 35 nM of ON-TARGETplus smartpool human CBP, p300 or siGENOME Non-targeting siRNA pool (Dharmacon) respectively into U2OS cells. Whole cell extracts were made at $72 \mathrm{~h}$ post transfection and used for immunoblotting. In experiments where CBP and p300 knockdowns 
were done in combination with JQ1 treatment, 500nM of JQ1 or equal volume of DMSO was added $18 \mathrm{~h}$ prior to harvesting siRNA transfected cells.

\section{Reagents}

Antibodies used for either immunoprecipitation or immunoblotting were Anti BRD4 (H-250) :sc-48772 and anti-Ac Histone H4: sc-34263 (Santa Cruz Biotech), anti-Ac Histone H3K4 (\#07-539; Millipore), anti-Ac Histone H3 (\#06-599; Upstate), anti-Ac Histone H3K9, H3K14, H3K18, H3K27, H3K56, anti-Histone H3 (from \#9927 kit, Cell Signaling) and antiHistone H4 (F-9: Santa Cruz Biotech), anti-Ac Histone H4K5, H4K8, H4K12, and H4K16 (from \#17-211 kit, Millipore), anti-Tubulin (ab6046; Abcam), CBP (A-22: sc-369, Santa Cruz Biotech), p300 (3G230/NM-11, ab-14984, Abcam) and anti-Ac Histone H3K122 (ab33309; Abcam). The validation of specificity of the anti-Ac Histone H3K122 antibody (Lot \#436839 and \#GR14667-1) and other antibodies used in the study are provided on the manufacturers' websites. HAT inhibitors Anacardic acid, Curcumin and Garcinol (Biovision) and C646 (Sigma) were dissolved in dimethyl sulfoxide (DMSO) to make concentrated stock solutions. Stock solutions of all HAT inhibitors were stored at $-80^{\circ} \mathrm{C}$. The (+)-JQ1 inhibitor (BPS Bioscience) was similarly dissolved in DMSO and stored.

\section{Recombinant proteins and peptides}

Flag-tagged BRD4and flag-tagged dTAF1 were purified from Sf9 cells as described ${ }^{11}$. 6XHis-tagged wild type BRD4 and derived mutant proteins were purified using the Ni-NTA spin column kit (Qiagen) according to the manufacturer's instructions. Control extracts (Mock) from uninfected Sf9 cells were prepared similarly. The purity of the 6XHis-tagged BRD4 used in the study was assessed by Mass spectrometric analysis (RPLC-MS/MS; NCI Laboratory of Proteomics), confirming the absence of any known HAT in the sample. GSTtagged hBRD4 was expressed and purified from E.coli cells (BL21 Rosetta-gami strain, Novagen) by inducing expression using $0.8 \mathrm{mM}$ IPTG overnight at $18^{\circ} \mathrm{C}$ and repeated pulldowns and concentration using GST Sepharose beads (Amersham pharmacia). Purified proteins were concentrated using microcon size exclusion columns (Millipore), recovered in HKEG buffer (20mM Hepes, pH 7.9, 100mM KCl, 0.2mM EDTA, 20\% vol/vol Glycerol) and stored frozen at $-80^{\circ} \mathrm{C}$. Highly purified (> 99\%) human histone H1.0, H1.4, H2A, H2B, H3, H4 and H3.1, H3.2, H3.3 substrates were from New England Biolabs as recombinant proteins expressed in E.coli; their purity was confirmed by the company through Mass Spectrometry Analysis (ESI-TOF MS) and peptide sequencing. Synthetic Histone H4 peptide (1-21) and Histone H3 peptide (1-21) were from SignalChem. Synthetic Histone H3 (1-20) peptide, Histone H3 (1-19); H3K14(Ac) peptide and Histone H3 (1-20); H3K9(Ac) peptides were from AnaSpec. Recombinant p300, expressed and purified from Sf9, with purity confirmed at $>95 \%$, was from ProteinOne.

\section{Immunoprecipitation and Immunoblots}

Whole cells extracts were made from transfected cells using the tissue extraction reagent (Invitrogen) as per the manufacturer's instructions. All immunoblot analyses were performed using secondary antibodies from Li-Cor and the Odyssey ${ }^{\mathrm{TM}}$ infrared scanner. Specific antibodies were as indicated in the figures. BRD4 and BRD4 mutant binding to histones was detected by first subjecting equimolar amounts $(0.6 \mu \mathrm{g})$ of 6 XHis-tagged 
BRD4 proteins and $1 \mu \mathrm{g}$ of histone $\mathrm{H} 3$ to a $\mathrm{HAT}$ assay in the presence or absence of $0.5 \mathrm{mM}$ Acetyl CoA for $30 \mathrm{~min}$ at $30^{\circ} \mathrm{C}$. Subsequently, the reaction was incubated for $2 \mathrm{~h}$ at $4{ }^{\circ} \mathrm{C}$ with equal amounts of Ni-NTA agarose beads to pull down BRD4 proteins with associated histones. Bead-bound proteins were washed twice with 50mM Tris (pH 8.0), $100 \mathrm{mM} \mathrm{NaCl}$, and $0.1 \%$ NP-40 and immunoblotted with both anti-BRD4 and anti-histone H3 antibodies to detect bead-bound BRD4 and associated histone H3.

\section{Solution HAT assays}

HAT assays were as described with minor changes ${ }^{38}$. Purified BRD4 (500 ng), TAF1 (625 ng) or p300 (750 ng) was incubated with $1 \mu \mathrm{g}$ histone substrate (New England Biolabs), 70 nCi of $\left[{ }^{3} \mathrm{H}\right]$ acetyl-CoA $(10 \mathrm{Ci} / \mathrm{pmol})$ and $15 \mathrm{nCi}$ of $\left[{ }^{14} \mathrm{C}\right]$ acetyl-CoA $(60 \mathrm{mCi} / \mathrm{pmol})$ in HAT buffer (10 mM butyric acid, $50 \mathrm{mM}$ Tris pH 8.0, $1 \mathrm{mM}$ DTT, and 0.1 mM EDTA) at $30^{\circ} \mathrm{C}$ for $30 \mathrm{~min}$. HAT inhibitors were included in the reaction mix where indicated. The reaction was stopped with SDS sample buffer; samples were run on $15 \%$ SDSpolyacrylamide gels, fixed overnight, dried and exposed to a tritium phosphoimager screen. Histone acetylation was analyzed using Storm PhosphoImager with the ImageQuant software. HAT assays analyzed by immunoblots were done as described above, but with 0.6 $\mathrm{mM}$ unlabeled Acetyl CoA (Sigma), and immunoblotted with the appropriate antibody.

\section{Filter binding HAT assay}

Filter binding HAT assay was as described ${ }^{39}$, and analyzed by capturing the acetylated products on Whatman filter paper. After stringent washes to remove residual free acetyl CoA, acetylation levels were quantified using a Microbeta Trilux scintillation counter.

\section{In-gel HAT assay}

The In-gel HAT assay was as described ${ }^{40}$ with modifications as follows. Three sets of $3 \mu \mathrm{g}$ purified rBRD4, hBRD4 and mock Sf9 extract were run on a 6\% SDS-PAGE gel containing $1 \mathrm{mg} / \mathrm{ml} \mathrm{H} 4$ peptide. The gel was cut into three strips, each with one set of proteins each. One set was denatured with $8 \mathrm{M}$ Urea for $1 \mathrm{hr}$ and renatured for $16 \mathrm{~h}$, following which the gel strip was soaked in HAT buffer containing $2.5 \mathrm{mM}$ Acetyl CoA and $1 \mathrm{mM}$ sodium butyrate for $1.5 \mathrm{~h}$ at $30^{\circ} \mathrm{C}$. The gel was washed $3 \mathrm{X}$ in $5 \%$ TCA to remove unbound Acetyl CoA, further washed $3 \mathrm{X}$ with Tris-glycine-SDS buffer, and immunoblotted with an anti-AcH4 antibody. This immunoblot was aligned with the other two gel strips containing BRD4 and mock extract that were silver stained or immunoblotted with anti-BRD4 antibody.

\section{In vitro Nucleosome assembly}

Purified 5S rDNA (208bp) was from New England Biolabs (NEB). Unmodified recombinant human nucleosomes were assembled on the 5S rDNA with purified human histone $\mathrm{H} 2 \mathrm{~A} / \mathrm{H} 2 \mathrm{~B}$ dimers and histone $\mathrm{H} 3 / \mathrm{H} 4$ tetramers using the EpiMark Nucleosome Assembly kit (NEB) following the manufacturer's instructions.

\section{Immunofluorescence analysis}

Cells were plated on cover slips and transfected with either a vector control or BRD4, with the addition of $5 \mathrm{mM}$ sodium butyrate. $18 \mathrm{~h}$ post-transfection, cells were fixed with $4 \%$ 
parafamaldeyhde, permeabilized with $0.5 \%$ Triton-X, and labeled with antibodies specific to BRD4, FLAG, AcH3K9 or AcH3K122 and stained with DAPI. Confocal image z-stacks were acquired using a Zeiss LSM510 META confocal microscope using a 63x planapochromat (N.A. 1.4) objective lens, an optical slice thickness of 1.0 um, a x-y pixel size of $0.349 \mathrm{um}$, and a z-step size of $0.5 \mathrm{um}$. Resultant image z-stacks were analyzed using the Surpass module of Imaris (v. 7.7.1) software. Nuclear volume measurements of cells were obtained based on DAPI staining using Imaris x64 7.6.0.

\section{Mass spectrometric analysis of acetylated histone H3.3}

Human histone H3.3 was subjected to HAT assays with p300, BRD4 or no enzyme (control). The acetylated histone bands were excised from a coommassie blue stained SDS-PAGE gel and subjected to in-gel tryptic digestion at $37^{\circ} \mathrm{C}$ overnight. Digested peptide samples were desalted by C18 ZipTip (Millipore), lyophilized and re-suspended in $16 \mu \mathrm{l}$ of $0.1 \%$ formic acid for LC-MS analysis. Sample $(6 \mu$ l) were loaded on an Easy LC 1000 nano-capillary HPLC system (Thermo Scientific) with a $15 \mathrm{~cm}$ Acclaim ${ }^{\circledR}$ PepMap ${ }^{\text {TM }}$ RSLC C18 column (Thermo Scientific) connected to an electrospray ionization (ESI) emitter, coupled online with a dual-pressure linear ion trap (LTQ Velos Pro) mass spectrometer (Thermo Scientific) for $\mu$ RPLC-MS/MS analysis. Peptides were eluted using a linear gradient of $2 \%$ mobile phase B (acetonitrile with $0.1 \%$ formiac acid) to $42 \%$ mobile phase B within $45 \mathrm{~min}$ at a constant flow rate of $200 \mathrm{~nL} / \mathrm{min}$. The fifteen most intense molecular ions in the MS scan were sequentially selected for collision-induced dissociation (CID) using a normalized collision energy of 35\%. The mass spectra were acquired at the mass range of $\mathrm{m} / \mathrm{z} 380$ 2000. The Easy Nano Spray ion source (Thermo Scientific) capillary voltage and temperature were set at $1.7 \mathrm{kV}$ and $275{ }^{\circ} \mathrm{C}$, respectively. The dynamic exclusion function on the mass spectrometer was enabled during the MS2 data acquisition. The MS/MS data were searched against the human histone H3.3 protein sequence using Proteome Discoverer 1.4 interfaced with SEQUEST HT (Thermo Scientific). Lysine acetylation (42.01 Da) and methionine oxidation (15.99 Da), were set as dynamic modifications. Up to two missed tryptic cleavage sites was allowed during the database search. The cut-off for legitimate identifications were: charge state dependent cross correlation $\left(\mathrm{X}_{\text {corr }}\right) \geq 2.0$ for $[\mathrm{M}+\mathrm{H}]^{1+}, \geq$ 2.5 for $[\mathrm{M}+2 \mathrm{H}]^{2+}$ and $\geq 3.0$ for $[\mathrm{M}+3 \mathrm{H}]^{3+}$. The results were normalized by deducting the acetylated lysines detected in the no-enzyme control sample from those detected in the BRD4 and p300 acetylated samples.

\section{Nucleosome dissociation assays}

5S rDNA (7.7 pmoles) was end labelled with 20 pmoles of $\gamma^{32} \mathrm{P}$ labeled ATP using T4 polynucleotide kinase (NEB) according to the manufacturer's protocol. Nucleosomes were assembled in vitro on radiolabeled 5S rDNA as described above. Labeled nucleosomes were subjected to HAT assays as described above. The extent of nucleosome eviction was analyzed by $6 \%$ polyacrylamide gel electrophoresis under native conditions in TBE buffer; the dried gel was exposed to a phosphorimager screen. For nucleosome eviction from native chromatin, native chromatin was prepared from U2OS cells using the ChromaFlash chromatin extraction kit (Epigentek). Extracted native chromatin was subjected to HAT assays as described. Nucleosome eviction was analyzed by $1 \%$ agarose gel electrophoresis and staining with ethidium bromide. 


\section{Nucleosome occupancy analysis}

Relative nucleosome occupancy analysis in U2OS cells transfected with a vector control, WT hBRD4, or the hBRD4 $\Delta 40$ aa-NT CoA Bs double mutant was performed as described previously ${ }^{41}$. Nuclei were isolated from transfected cells and digested with $100 \mathrm{U}$ of MNase per $100 \mathrm{ul}$ reaction at room temperature for 5, 10, or $15 \mathrm{~min}$. Mononucleosomal-sized DNA was purified from the sample showing almost complete digestion and subjected to real-time PCR with primer sets designed to span the regions of interest (Supplementary Table 2). To account for differences in primer efficiency, the data were normalized to the vector control at each point.

\section{ChIP-Seq analysis}

All ChIP-seq datasets used in this study were downloaded from public repositories. BRD4, AcH3K122 ChIP-seq data sets for MCF-7 cells were from the NCBI Gene Expression Omnibus database as raw fastq files (accession numbers GSE35954 and GSE55923; ${ }^{20,42}$. p300/CBP ChIP-seq data for MCF-7 cells were from the ArrayExpress ChIP-seq database (accession number E-MTAB-785). CTCF, MYC and Gata3 ChIP-seq data sets for MCF-7 cells were from the ENCODE project (The ENCODE Project Consortium 2012) at the UCSC Genome Browser. BRD4 and H3K27ac ChIP-seq datasets for diffuse large B cell lymphoma cell line (LY1) in the presence of JQ1 or DMSO were from NCBI GEO (accession number: GSE46663, ${ }^{43}$ ). BRD4, H3ac and H4ac ChIP-seq datasets for $1 \mathrm{~h} \mathrm{LPS}$ stimulated mouse bone marrow-derived macrophages in the presence of iBET BRD4 inhibitor or DMSO were from NCBI GEO (accession number: GSE21910 21). All ChIP-seq datasets were aligned using Bowtie ${ }^{44}$ to the reference genome (hg 19 for human datasets and $\mathrm{mm} 10$ for mouse datasets). Only reads mapped uniquely to the genome were used for the downstream analysis. Peaks for ChIP-seq data were identified using HOMER (http:// homer.salk.edu/homer/). AcH3K122 enrichment was calculated based on the center of each peak of BRD4 and the other transcription factors. The average profile plots at TSSs were generated using ngsplot (https://github.com/shenlab-sinai/ngsplot).

\section{MNase-Seq analysis}

MNase-seq analysis to measure genome-wide nucleosome occupancy was done as described previously ${ }^{45}$. Briefly, nuclei from 10-20 million cells were subjected to MNase digestion to obtain a majority of mononucleosomes (>80\% as estimated on an E-gel system). DNA from the mononuclesomes was purified and prepared for sequencing by repairing ends, adapter ligation, size selection and amplification using Illumina PCR primers. Purified DNA was used directly for cluster generation and sequencing analysis on a Genome Analyzer II (Illumina) following manufacturers' protocols. Sequenced reads of mostly $126 \mathrm{bp}$ were mapped to the human genome (hg19) reference genomes using BWA-MEM ${ }^{46}$ and all duplicated reads were removed. Nucleosome profiles were obtained using DANPOS ${ }^{47}$. DNA fragment size was determined by the distance between the paired-end reads and read length was adjusted to be half of the nucleosome size (74 bp). Nucleosome occupancy was calculated relative to the Transcription Start Sites (TSS) based on the RefSeq gene annotation. Average nucleosome occupancy for $2 \mathrm{k}$ upstream and $2 \mathrm{k}$ downstream of each 
TSS was also calculated to determine the differential nucleosome occupancy between upstream and downstream of TSS.

\section{RNA-seq analysis}

RNA-seq analysis was done as described before ${ }^{48}$. mRNAs isolated from control and BRD4 transfected were converted to cDNA using standard protocols. The cDNAs were fragmented to $100-200 \mathrm{bp}$ using sonication, followed by end repair and Solexa adaptor ligation. The products were sequenced on an Illumina GAII system according to established procedures. Reads were aligned the human genome (hg19) reference genomes using Tophat ${ }^{49}$. RPKM values were calculated using SeqGene package ${ }^{50}$. The correlation between RNA expression levels and nucleosome occupancy was determined by using a PERL script, which will be made available upon request. $P$ values were calculated using chi square test, comparing wild type and mutant BRD4, across all bins.

\section{mRNA quantification by qRT-PCR}

U2OS cells were transfected with either BRD4 WT, BRD4 HAT mutant or vector only and cultured for 18 hours in the presence of $5 \mathrm{mM}$ sodium butyrate. Total RNA was isolated using TRIzol reagent (Invitrogen) following the manufacturer's instructions. Reverse transcription was performed in a $20-\mu$ reaction system containing $1.5 \mu \mathrm{g}$ of total RNA using oligo(dT) primer (Invitrogen) and the Thermoscript RT-PCR system (Invitrogen) following the manufacturer's instructions. Real time PCR using gene-specific primers was performed in triplicate using $1 \mu \mathrm{l}$ of RT product in a $25-\mu \mathrm{l}$ reaction containing $12.5 \mu \mathrm{l}$ of iQ SYBR Green supermix (Bio-Rad) and $0.4 \mu \mathrm{m}$ of each primer. mRNA levels of each gene was normalized to GAPDH mRNA level. Primer sequences are shown in supplementary Table 2.

\section{JQ1 treatment of cells and ChIP analysis}

U2OS cells were treated with either JQ1 or DMSO vehicle for $18 \mathrm{~h}$ and harvested for either immunoblots or ChIP analysis. For ChIP-qPCR, cells $\left(3 \times 10^{6}\right)$ were cross-linked with $1 \%$ formaldehyde for $15 \mathrm{~min}$ at room temperature and sheared (1\% SDS, 45 minutes, 100\% amplitude) in a sonicator (Qsonica) to produce $\sim 300 \mathrm{bp}$ fragments. Lysates were subjected to immunoprecipitation with Dynabeads Protein G-coupled magnetic beads (Life Technologies) after retaining $10 \%$ as input. Recovered DNA was subjected to qPCR with locus specific primer pairs. The results were normalized to the input DNA.

\section{Development of BRD4 KO mice and mouse embryonic fibroblasts}

The BRD4 knockout allele (BRD4--) and the BRD4 floxed allele (BRD4f) were both designed to remove the third exon (carrying the ATG) of BRD4. The $B R D 4^{--}$allele was generated by replacing a $2 \mathrm{~kb}$ genomic region containing exon 3 with an exogenous $6.6 \mathrm{~Kb}$ fragment carrying the geo sequence. In the BRD4f allele, exon 3 is surrounded by 2 LoxP sites. $B R D 4^{+-}$mice were bred with mice expressing the Cre recombinase under the control of the LCK proximal promoter (Taconic). $B R D 4^{+/-} \mathrm{LCK}^{-} \mathrm{Cr}^{+/-}$offspring were then bred with $B R D 4 \mathrm{f} / \mathrm{f}$ mice to produce either $B R D 4 \mathrm{f}^{--} L C K-C r e^{+/-}$(BRD4 KO) or $B R D 4 \mathrm{f}^{\prime+} L C K-$ $\mathrm{Cr}^{+/-}$(BRD4 Het) mice. Cells were prepared from thymus, counted and assessed for CD4, CD8 surface protein expression by flow cytometry using a FACS Aria. Mus musculus, 
C57/BL6 BRD4 Flox ${ }^{+/-}$- LCK-CRE ${ }^{+/}$, both male and female mice 8 weeks old were used. No blinding or randomization was used in the animal studies. Fig 7c FACs profile is representative of 2 male and 5 female 8 week old mice analyzed in seven independent experiments. Fig. 7c lower panels are representative of 2 male mice of each genotype, assayed in two independent experiments. All experiments with mice in the study are in compliance with the NIH animal care and use committee, approved under protocol \# EIB-076. Mouse embryonic fibroblasts were generated from the BRD4 KO mice. Cells were co-infected with Cre-GFP and either BRD4-NGFR or BRD4 $\triangle 40$ N-C-CoA Bs-NGFR. GFPpositive cells were sorted by FACS to generate stable MEF cell lines.

\section{Supplementary Material}

Refer to Web version on PubMed Central for supplementary material.

\section{Acknowledgments}

We thank D. Levens, E. Harlow, R. Sen and L. Staudt for critical reading of the manuscript and members of the Singer lab for discussions. We are especially grateful to K. Placek and K. Zhao for their technical help with the RNA-seq experiments. We thank M. Zhou for the mass spectrometry analysis and the NIH CIT/Biowulf cluster used in the analyses of the ChIP-seq data. This research was supported by the Intramural Research Program of the NIH, National Cancer Institute, Center for Cancer Research.

\section{References}

1. Wu SY, Chiang CM. The double bromodomain-containing chromatin adaptor Brd4 and transcriptional regulation. J Biol Chem. 2007; 282:13141-5. [PubMed: 17329240]

2. Dey A, et al. A bromodomain protein, MCAP, associates with mitotic chromosomes and affects G(2)-to-M transition. Mol Cell Biol. 2000; 20:6537-49. [PubMed: 10938129]

3. Floyd SR, et al. The bromodomain protein Brd4 insulates chromatin from DNA damage signalling. Nature. 2013; 498:246-50. [PubMed: 23728299]

4. Dey A, Nishiyama A, Karpova T, McNally J, Ozato K. Brd4 marks select genes on mitotic chromatin and directs postmitotic transcription. Mol Biol Cell. 2009; 20:4899-909. [PubMed: 19812244]

5. Kadauke S, Blobel GA. Mitotic bookmarking by transcription factors. Epigenetics Chromatin. 2013; 6:6. [PubMed: 23547918]

6. Mochizuki K, et al. The bromodomain protein Brd4 stimulates G1 gene transcription and promotes progression to S phase. J Biol Chem. 2008; 283:9040-8. [PubMed: 18223296]

7. Wu SY, Lee AY, Lai HT, Zhang H, Chiang CM. Phospho switch triggers Brd4 chromatin binding and activator recruitment for gene-specific targeting. Mol Cell. 2013; 49:843-57. [PubMed: 23317504]

8. You J, et al. Regulation of aurora B expression by the bromodomain protein Brd4. Mol Cell Biol. 2009; 29:5094-103. [PubMed: 19596781]

9. Loven J, et al. Selective inhibition of tumor oncogenes by disruption of super-enhancers. Cell. 2013; 153:320-34. [PubMed: 23582323]

10. Chiang CM. Brd4 engagement from chromatin targeting to transcriptional regulation: selective contact with acetylated histone H3 and H4. F1000 Biol Rep. 2009; 1:98. [PubMed: 20495683]

11. Devaiah BN, et al. BRD4 is an atypical kinase that phosphorylates serine 2 of the RNA polymerase II carboxy-terminal domain. Proc Natl Acad Sci U S A. 2012; 109:6927-32. [PubMed: 22509028]

12. Devaiah BN, Singer DS. Cross-talk among RNA polymerase II kinases modulates C-terminal domain phosphorylation. J Biol Chem. 2012; 287:38755-66. [PubMed: 23027873]

13. Struhl K. Histone acetylation and transcriptional regulatory mechanisms. Genes Dev. 1998; 12:599-606. [PubMed: 9499396] 
14. Fukuda H, Sano N, Muto S, Horikoshi M. Simple histone acetylation plays a complex role in the regulation of gene expression. Brief Funct Genomic Proteomic. 2006; 5:190-208. [PubMed: 16980317]

15. Sterner DE, Berger SL. Acetylation of histones and transcription-related factors. Microbiol Mol Biol Rev. 2000; 64:435-59. [PubMed: 10839822]

16. Kouzarides T. Chromatin modifications and their function. Cell. 2007; 128:693-705. [PubMed: 17320507]

17. Strahl BD, Allis CD. The language of covalent histone modifications. Nature. 2000; 403:41-5. [PubMed: 10638745]

18. Dey A, Chitsaz F, Abbasi A, Misteli T, Ozato K. The double bromodomain protein Brd4 binds to acetylated chromatin during interphase and mitosis. Proc Natl Acad Sci U S A. 2003; 100:875863. [PubMed: 12840145]

19. Tropberger P, Schneider R. Scratching the (lateral) surface of chromatin regulation by histone modifications. Nat Struct Mol Biol. 2013; 20:657-61. [PubMed: 23739170]

20. Tropberger $P$, et al. Regulation of transcription through acetylation of H3K122 on the lateral surface of the histone octamer. Cell. 2013; 152:859-72. [PubMed: 23415232]

21. Nicodeme E, et al. Suppression of inflammation by a synthetic histone mimic. Nature. 2010; 468:1119-23. [PubMed: 21068722]

22. Nishiyama A, Dey A, Miyazaki J, Ozato K. Brd4 is required for recovery from antimicrotubule drug-induced mitotic arrest: preservation of acetylated chromatin. Mol Biol Cell. 2006; 17:814-23. [PubMed: 16339075]

23. Kruhlak MJ, et al. Regulation of global acetylation in mitosis through loss of histone acetyltransferases and deacetylases from chromatin. J Biol Chem. 2001; 276:38307-19. [PubMed: 11479283]

24. Zhao R, Nakamura T, Fu Y, Lazar Z, Spector DL. Gene bookmarking accelerates the kinetics of post-mitotic transcriptional re-activation. Nat Cell Biol. 2011; 13:1295-304. [PubMed: 21983563]

25. Wang R, You J. Mechanistic analysis of the role of bromodomain-containing protein 4 (BRD4) in BRD4-NUT oncoprotein-induced transcriptional activation. J Biol Chem. 2015; 290:2744-58. [PubMed: 25512383]

26. Dutnall RN, Tafrov ST, Sternglanz R, Ramakrishnan V. Structure of the histone acetyltransferase Hat1: a paradigm for the GCN5-related N-acetyltransferase superfamily. Cell. 1998; 94:427-38. [PubMed: 9727486]

27. Filippakopoulos P, et al. Selective inhibition of BET bromodomains. Nature. 2010; 468:1067-73. [PubMed: 20871596]

28. Vernarecci S, Tosi F, Filetici P. Tuning acetylated chromatin with HAT inhibitors: a novel tool for therapy. Epigenetics. 2010; 5:105-11. [PubMed: 20160510]

29. McBrian MA, et al. Histone acetylation regulates intracellular pH. Mol Cell. 2013; 49:310-21. [PubMed: 23201122]

30. Zheng Y, Thomas PM, Kelleher NL. Measurement of acetylation turnover at distinct lysines in human histones identifies long-lived acetylation sites. Nat Commun. 2013; 4:2203. [PubMed: 23892279]

31. Devaiah BN, Singer DS. Two faces of brd4: mitotic bookmark and transcriptional lynchpin. Transcription. 2013; 4:13-7. [PubMed: 23131666]

32. Baranello, et al. RNA Polymerase II regulates Topoisomerase 1 activity to favor efficient transcription. Cell. 2016. http://dx.doi.org/10.1016/j.cell.2016.02.036

33. Delmore JE, et al. BET bromodomain inhibition as a therapeutic strategy to target c-Myc. Cell. 2011; 146:904-17. [PubMed: 21889194]

34. Zhang W, et al. Bromodomain-containing protein 4 (BRD4) regulates RNA polymerase II serine 2 phosphorylation in human CD4+ T cells. J Biol Chem. 2012; 287:43137-55. [PubMed: 23086925]

35. Wang R, Li Q, Helfer CM, Jiao J, You J. Bromodomain protein Brd4 associated with acetylated chromatin is important for maintenance of higher-order chromatin structure. J Biol Chem. 2012; 287:10738-52. [PubMed: 22334664] 
36. Jones MJ, Jallepalli PV. Chromothripsis: chromosomes in crisis. Dev Cell. 2012; 23:908-17. [PubMed: 23153487]

37. Liu W, et al. Brd4 and JMJD6-associated anti-pause enhancers in regulation of transcriptional pause release. Cell. 2013; 155:1581-95. [PubMed: 24360279]

38. Devaiah BN, et al. Novel functions for TAF7, a regulator of TAF1-independent transcription. J Biol Chem. 2010; 285:38772-80. [PubMed: 20937824]

39. Berndsen CE, Denu JM. Assays for mechanistic investigations of protein/histone acetyltransferases. Methods. 2005; 36:321-31. [PubMed: 16085424]

40. Mizzen CA, et al. The TAF(II)250 subunit of TFIID has histone acetyltransferase activity. Cell. 1996; 87:1261-70. [PubMed: 8980232]

41. Kotekar AS, Weissman JD, Gegonne A, Cohen H, Singer DS. Histone modifications, but not nucleosomal positioning, correlate with major histocompatibility complex class I promoter activity in different tissues in vivo. Mol Cell Biol. 2008; 28:7323-36. [PubMed: 18809568]

42. Nagarajan S, et al. Bromodomain protein BRD4 is required for estrogen receptor-dependent enhancer activation and gene transcription. Cell Rep. 2014; 8:460-9. [PubMed: 25017071]

43. Chapuy B, et al. Discovery and characterization of super-enhancer-associated dependencies in diffuse large B cell lymphoma. Cancer Cell. 2013; 24:777-90. [PubMed: 24332044]

44. Langmead B, Salzberg SL. Fast gapped-read alignment with Bowtie 2. Nat Methods. 2012; 9:3579. [PubMed: 22388286]

45. Cui K, Zhao K. Genome-wide approaches to determining nucleosome occupancy in metazoans using MNase-Seq. Methods Mol Biol. 2012; 833:413-9. [PubMed: 22183607]

46. Li H. Aligning sequence reads, clone sequences and assembly contigs with BWA-MEM. 2013 arXiv:1303.3997.

47. Chen K, et al. DANPOS: dynamic analysis of nucleosome position and occupancy by sequencing. Genome Res. 2013; 23:341-51. [PubMed: 23193179]

48. Wei G, Hu G, Cui K, Zhao K. Genome-wide mapping of nucleosome occupancy, histone modifications, and gene expression using next-generation sequencing technology. Methods Enzymol. 2012; 513:297-313. [PubMed: 22929775]

49. Trapnell C, Pachter L, Salzberg SL. TopHat: discovering splice junctions with RNA-Seq. Bioinformatics. 2009; 25:1105-11. [PubMed: 19289445]

50. Deng X. SeqGene: a comprehensive software solution for mining exome- and transcriptomesequencing data. BMC Bioinformatics. 2011; 12:267. [PubMed: 21714929] 
a

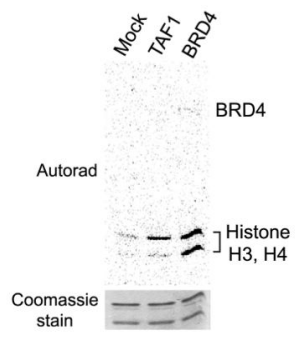

b

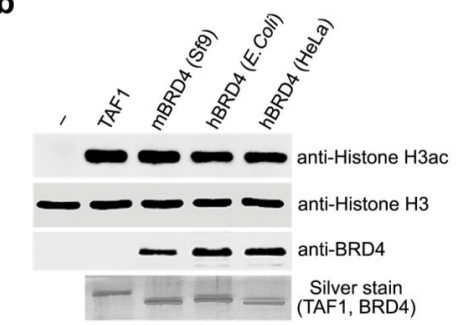

c

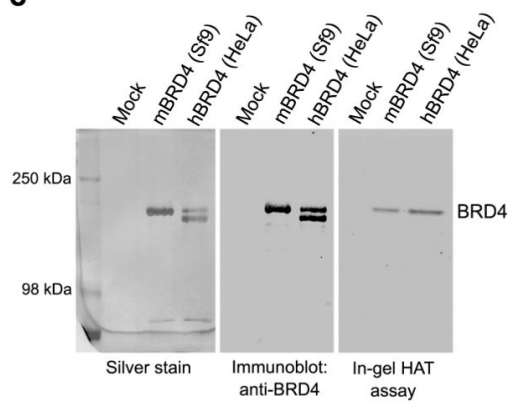

d

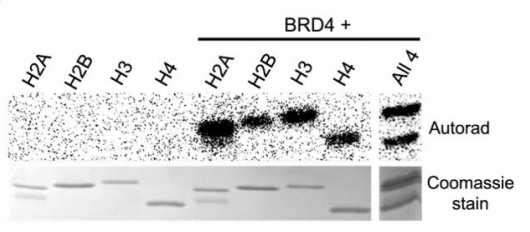

e

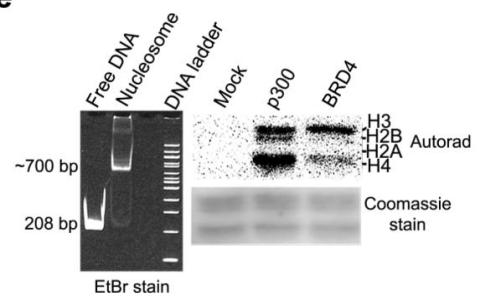

f

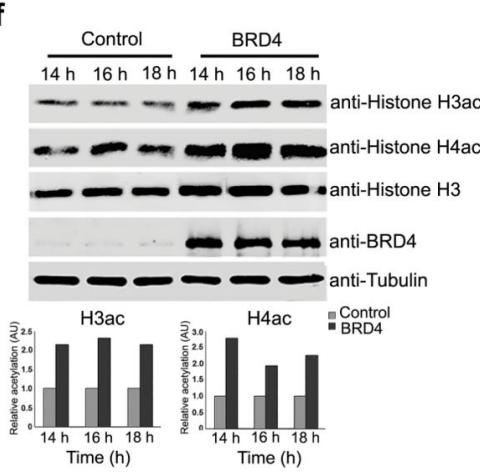

Fig. 1. BRD4 has intrinsic histone acetyltransferase activity

(a) HAT assays of $\mathrm{H} 3$ and $\mathrm{H} 4$ (1 $\mu \mathrm{g})$ with radiolabeled AcCoA with recombinant BRD4 (500 ng), mTAF1 (250 ng) or mock.

(b) HAT assays of rHis-mBRD4 $(500 \mu \mathrm{g})$ from insect sf9 cells, GST-hBRD4 from E.coli, Flag-hBRD4 from HeLa cells and rTAF1 with histone H3 $(1 \mu \mathrm{g})$ and acetyl CoA.

Immunoblotting was with anti-AcH3, anti-H3 or anti-BRD4 antibodies. Bottom panel, silver staining as a loading control.

(c) Recombinant mBRD4 from sf9 cells, hBRD4 (3.5 $\mu \mathrm{g})$ and control Sf9 extract resolved on a denaturing gel (left panel), immunoblotted with anti-BRD4 (middle panel) or subjected to an in-gel HAT assay; acetylated $\mathrm{H} 4$ peptide visualized with anti-AcH4 (right hand panel). Faster mobility band in hBRD4 lane is a degradation product.

(d) Histones H2A, H2B, H3, H4 (1 $\mu \mathrm{g})$ or all four (1 $\mu \mathrm{g}$ each) incubated with or without 500ng BRD4 in a HAT assay followed by autoradiography or coomassie staining.

(e) Mononucleosomes (equivalent to $700 \mathrm{bp}$ ) (10pmol) assembled on 5S rDNA (208 bp), visualized in ethidium bromide stained gels (left panel), subjected to a HAT assay with or without recombinant p300 (500 ng) or BRD4 (500 ng) with radiolabeled AcCoA and visualized by autoradiography or coomassie staining.

(f) Histone $\mathrm{H} 3$, H4ac and $\mathrm{H} 3 \mathrm{ac}$ immunoblots of whole cell extracts (WCE) from HeLa cells grown 14, 16 and 18hr following transfection with $3 \mu \mathrm{g}$ hBRD4 expression plasmid or control. AU: arbitrary units. Data are representative of 3 independent experiments. 
Uncropped images of gels and immunoblots are shown in Supplementary Data Set 1 
a

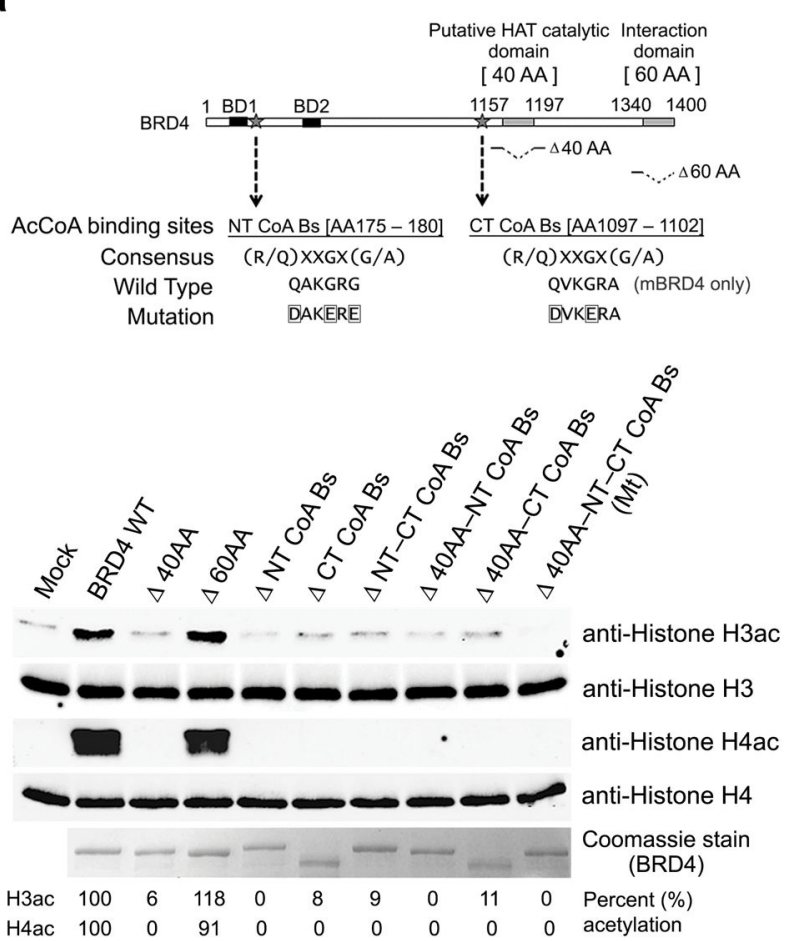

b
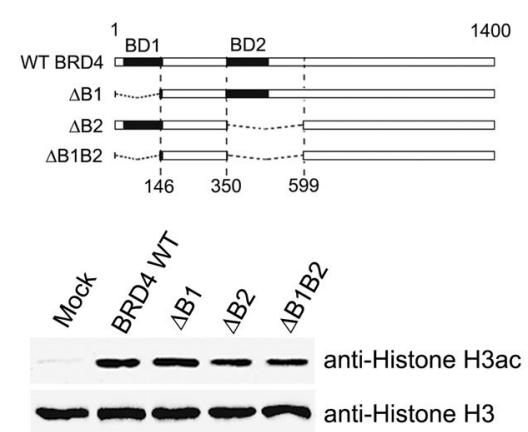

C

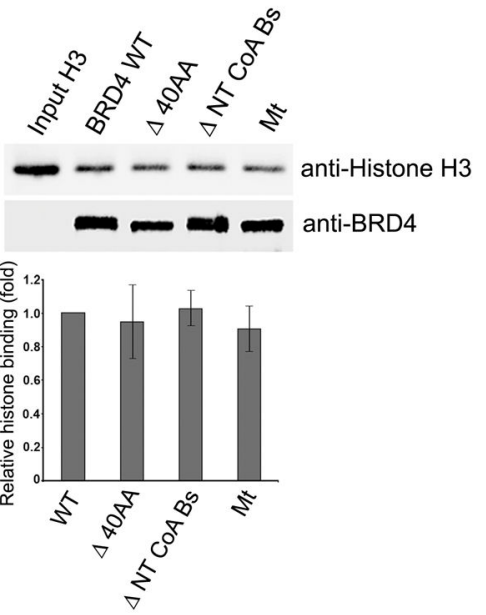

Fig. 2. BRD4 has discrete acetyl CoA binding and HAT catalytic sites

(a) Map (upper panel) shows putative acetyl CoA binding (stars), HAT catalytic site (shaded) and interaction domain (shaded) (upper). Amino acid substitutions and deletions are shown below the map. (lower panel) Mouse BRD4 (300ng) or equimolar amounts of BRD4 mutants used in HAT assays with $1 \mu \mathrm{g}$ histone $\mathrm{H} 3$ or $\mathrm{H} 4$ and subsequently immunoblotted with anti-AcH3,anti-AcH4 or anti-H3 and H4 antibodies. Densitometric quantification of acetylated histones, normalized to total histones, is shown below the immunoblots. The triple mutant, $\triangle 40 \mathrm{AA}-\mathrm{NT}-\mathrm{CT}$ CoA Bs, will be referred to as Mt hereafter. (b) BRD4 (300ng) or equimolar amounts of the BRD4 bromodomain deletion mutants, $\Delta \mathrm{B} 1$, $\Delta \mathrm{B} 2, \Delta \mathrm{B} 1 \mathrm{~B} 2$, (upper panel) used in HAT assays with $1 \mu \mathrm{g}$ histone $\mathrm{H} 3$ and subsequently immunoblotted with anti-AcH3 and anti-H3 antibodies (lower panel).

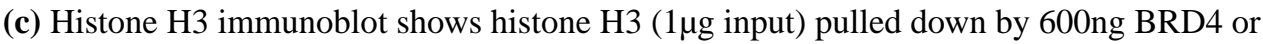
equimolar amounts of BRD4 HAT mutants. Graph shows relative binding efficiency. Error bars, s.e.m. ( $n=3$ independent experiments, $P<0.05$ by two-tailed student's $t$-test). Uncropped images of immunoblots are shown in Supplementary Data Set 1 
a

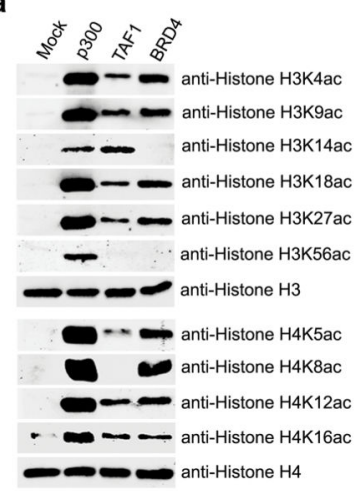

b

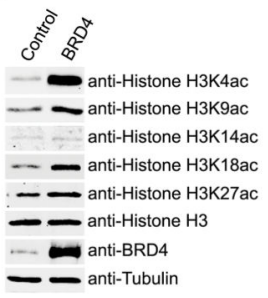

d
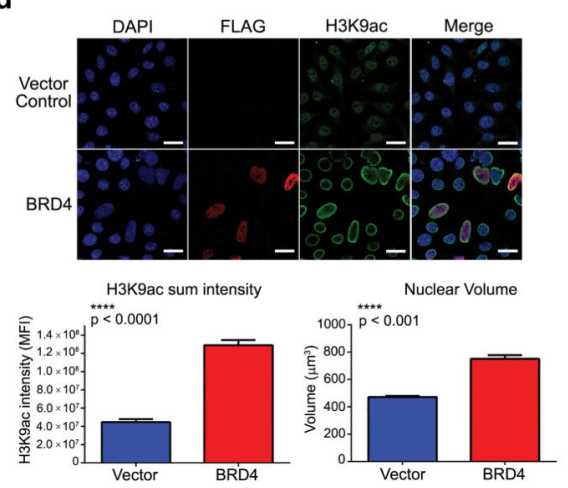

e c

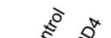

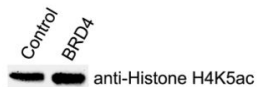

anti-Histone H4K8ac

- anti-Histone H4K12ac

- anti-Histone H4K16ac

- anti-Histone $\mathrm{H}_{4}$

- anti-BRD4

- anti-Tubulin

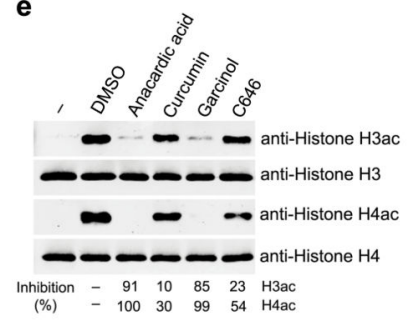

Fig. 3. BRD4 histone acetyltransferase activity is distinct from other HAT's

(a) Immunoblots, with antibodies specific for different acetylated lysine residues of histone $\mathrm{H} 3$ and $\mathrm{H} 4$, following in vitro HAT assays with $1 \mu \mathrm{g}$ histone $\mathrm{H} 3$ or $\mathrm{H} 4$ and $500 \mathrm{ng}$ of BRD4, $750 \mathrm{ng}$ of p300, $625 \mathrm{ng}$ of TAF1 (to achieve equimolar amounts) or no enzyme (Mock). (b and c) Immunoblots, with antibodies specific for different acetylated histone $\mathrm{H} 3$ (b) and H4 (c) lysine residues, of whole cell extracts (WCE) from HeLa cells transfected with $3 \mu \mathrm{g}$ pCMV2 vector (control) or hBRD4 (BRD4) at $18 \mathrm{hr}$ post-transfection in the presence of sodium butyrate.

(d) Representative images of HeLa cells transfected with $3 \mu \mathrm{g}$ pCMV2 (vector control) or FLAG-hBRD4, stained with DAPI, and analyzed by immunofluorescence with anti-FLAG and anti-AcH3K9 antibodies (upper panels); Scale bars, $20 \mu \mathrm{m}$. Intensity of H3K9ac staining (lower left panel) and nuclear volume, determined by automated analysis of the 3D volume of DAPI-stained nuclei (lower right panel), of transfected cells $(n=130)$ are plotted as histograms. Error bars represent \pm s.e.m.; $p$ values were based on two-tailed student's $t$ tests. Results shown are representative of three independent transfection experiments.

(e) Immunoblots of $1 \mu \mathrm{g}$ histones H3 and H4 subjected to HAT assays with 500ng BRD4 in the presence of $2 \%$ DMSO, $100 \mu \mathrm{M}$ Anacardic acid, $100 \mu \mathrm{M}$ Curcumin, $20 \mu \mathrm{M}$ Garcinol and $10 \mu \mathrm{M}$ C646. Inhibition (\%) of BRD4 HAT activity is indicated at the bottom.

Uncropped images of immunoblots are shown in Supplementary Data Set 1

Nat Struct Mol Biol. Author manuscript; available in PMC 2016 November 09. 


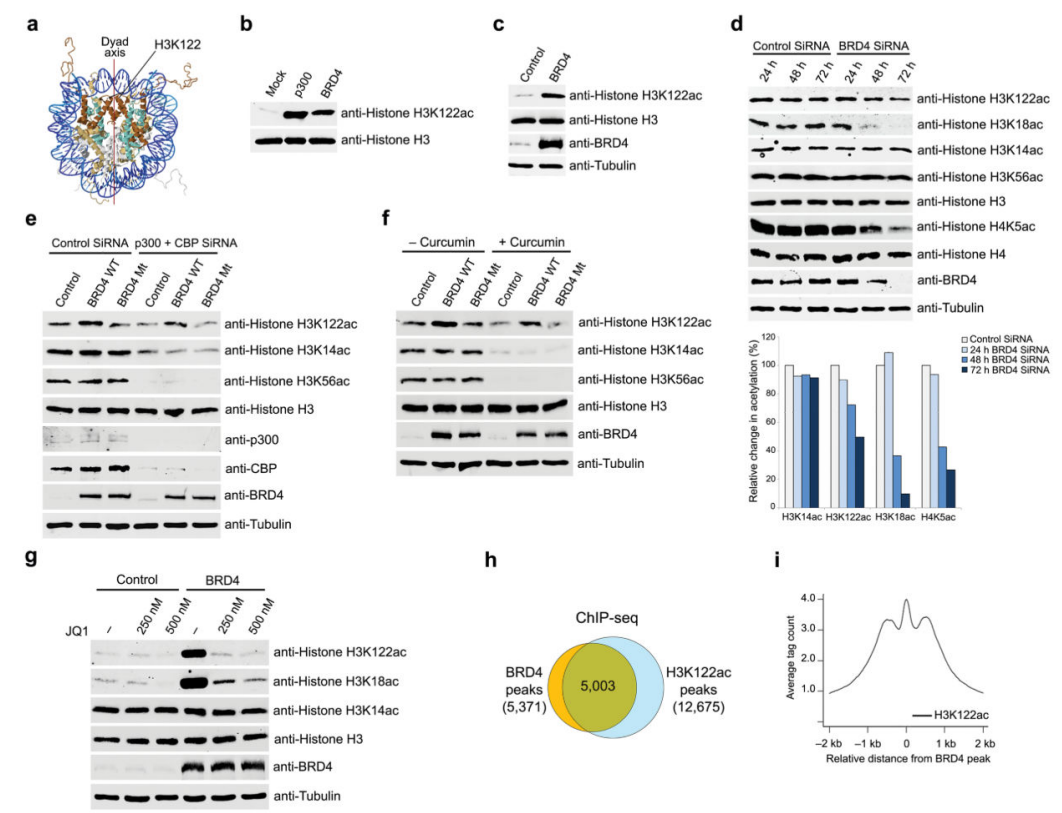

Fig. 4. BRD4 acetylates H3K122

(a) Nucleosome structure (PDB code 1KX5); H3K122 residues indicated at dyad axis.

(b) HAT assays with H3 (1ug) and 500ng p300, BRD4 or no enzyme (mock); immunoblotting as indicated.

(c) Immunoblots of HeLa WCE made $18 \mathrm{hr}$ post-transfection with $3 \mu \mathrm{g}$ hBRD4 or control plasmid.

(d) Immunoblots of WCE from HeLa cells isolated 24, 48 and 72hr post-transfection with BRD4 or control siRNA, as indicated. Acetylated histone levels quantified relative to total histone $\mathrm{H} 3$ and $\mathrm{H} 4$ at different intervals post-transfection (lower panel). Bars are average of two independent experiments.

(e) Immunoblots of WCE from U2OS cells transfected with p300+CBP or control siRNAs,

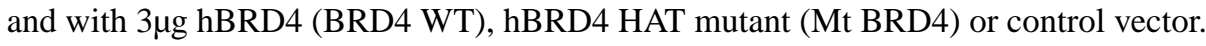
Results shown are representative of two independent experiments.

(f) Immunoblots, as indicated, of WCE fromU2OS cells transfected with $3 \mu \mathrm{g}$ BRD4 WT, Mt BRD4 or control plasmid, treated with $50 \mu \mathrm{M}$ curcumin or DMSO. Results shown are representative of two independent experiments.

(g) Immunoblots, as indicated, of WCE from U2OS cells, transfected with $3 \mu \mathrm{g}$ hBRD4 or control plasmid, and treated with JQ1 (250 and 500nM) or DMSO (-) for 18hr

(h) Metagene analysis showing genome-wide co-localization of BRD4 and H3K122ac in MCF-7 cells.

(i) Aggregation plot showing mean tag count for H3K122ac at BRD4 peaks. Uncropped images of immunoblots are shown in Supplementary Data Set 1 
a

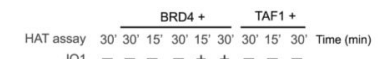

JQ1 - - - + + + - -

$\mathrm{ACCOA}+-++++++$

II 1 .
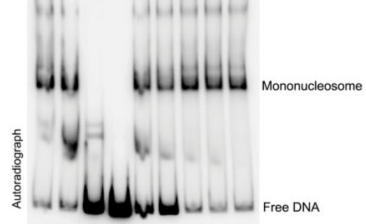

然 D -

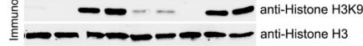

c

BRD4 (1) (2) (3) (4) (5) (6) (7)

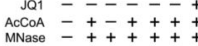

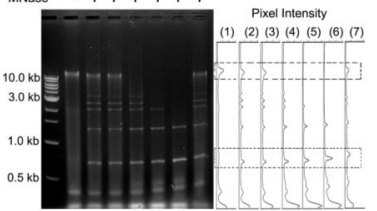

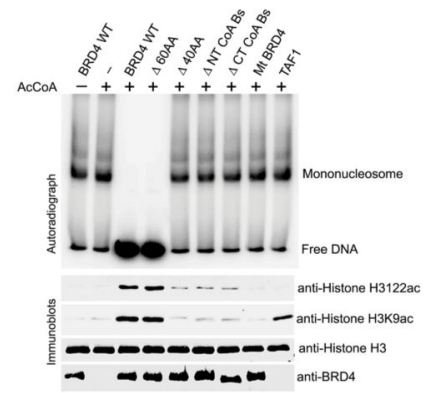

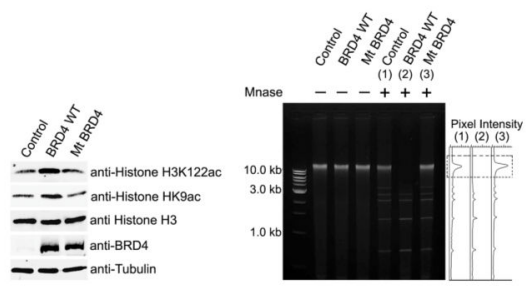

Fig. 5. BRD4 acetylation of H3K122 mediates histone eviction and nucleosome clearance a) Autoradiogram showing mononucleosomes (10 pmol) assembled in vitro with radiolabeled 5S rDNA and subjected to HAT assays for 15 and 30 minutes with or without $500 \mathrm{ng}$ BRD4 or TAF1 in the presence or absence of acetyl CoA or $500 \mu \mathrm{M}$ JQ1. Free DNA resolved from mononucleosomes by electrophoresis (upper panel). Immunoblots of identical HAT assays done in parallel with non-radiolabeled mononucleosomes, showing extent of acetylation of H3K122 and H3K9; histone H3 immunoblot served as control (lower panels).

(b) HAT assays and immunoblots as in (a) with or without BRD4, BRD4 HAT mutants or TAF1 (500ng).

(c) Ethidium Bromide stained agarose gels showing native chromatin isolated from U2OS cells that was incubated in HAT assays with or without increasing amounts of BRD4 (Lane 3: $1 \mu \mathrm{g}$; Lane 4, 5, 6: 0.2, 0.5, $1 \mu \mathrm{g}$ respectively; Lane 7: $1 \mu \mathrm{g})$, Acetyl CoA and $500 \mu \mathrm{M} \mathrm{JQ1}$ (lane 7) and then digested with 0.1U MNase for 3 min (left panel). Quantification of digested bands showing increased MNase digestion correlated with increasing BRD4 (right panel).

(d) Ethidium Bromide stained agarose gels showing native chromatin isolated from U2OS

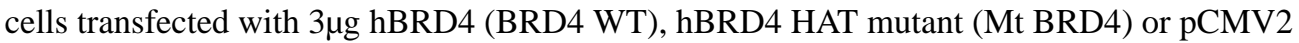
vector (control) in the presence of sodium butyrate and incubated with or without $0.1 \mathrm{U}$ MNase for 3 min (middle panel). Quantification of digested bands from cells transfected with vector (1), BRD4 WT (2) and Mt BRD4 (3) (right panel). Immunoblots of WCEs from the transfected cells showing H3K122ac, H3K9ac and BRD4 levels (left panel).

Uncropped images of gels and immunoblots are shown in Supplementary Data Set 1 

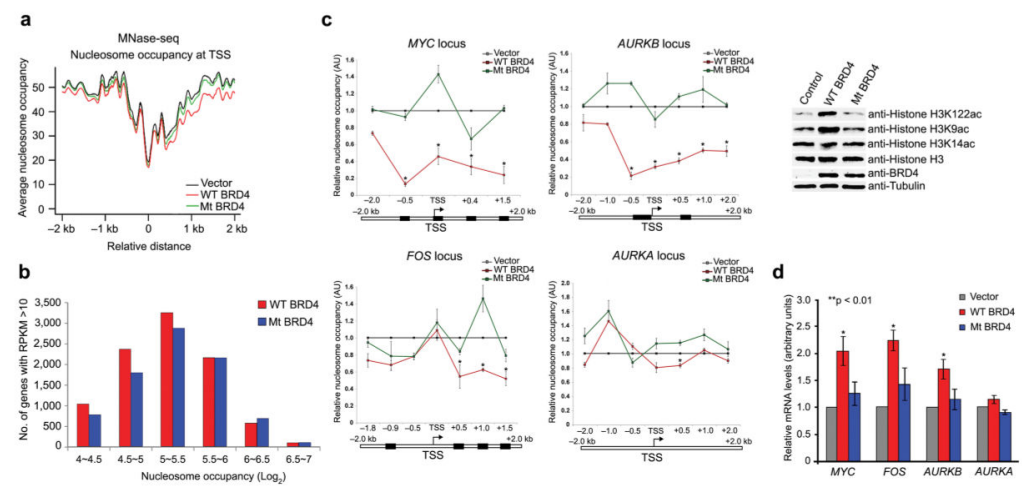

e
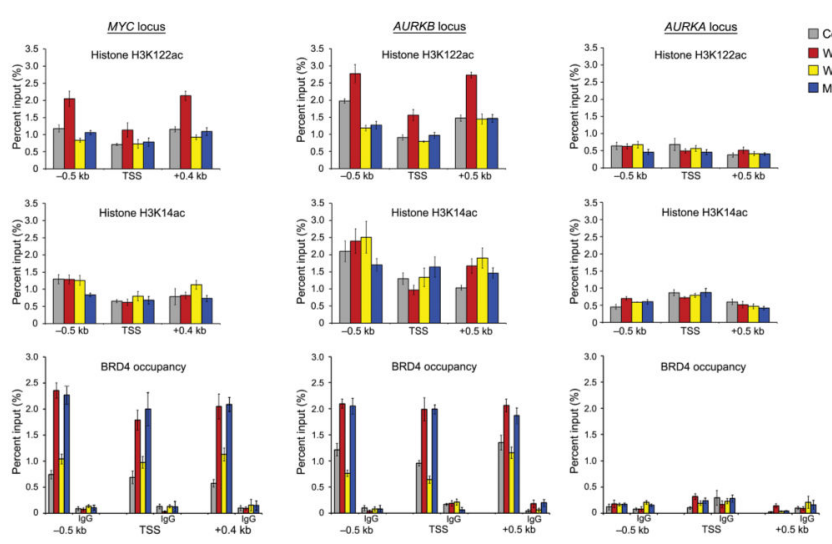

Fig. 6. BRD4 HAT activity decreases nucleosome occupancy genome-wide

(a) MNase-Seq plot showing average nucleosome occupancy relative to genome-wide TSS

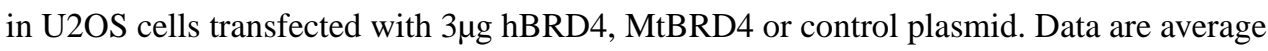
of two independent transfections.

(b) Graph showing RNA-seq of U2OS cells transfected as in (a) with number of individual transcript levels RPKM $>10$ plotted as a function of nucleosome occupancy within $2 \mathrm{~Kb}$ of the TSS. The difference between wild type (WT) and HAT mutant (MT) was statistically significant $\left(p=2.2^{\mathrm{e}-15}\right.$ by Chi-Square test; $n=4$ technical replicates from two independent experiments).

(c) Nucleosome occupancy following U2OS transfection with 3 ug hBRD4, Mt BRD4 or control plasmid. Line graphs represent nucleosome occupancy (Arbitrary Units; AU) relative to the vector control at each point (set as 1), at sites between -2.0 and $+2.0 \mathrm{~Kb}$ from TSS of MYC, AURKB, FOS and AURKA (see Supplementary Table 2 for list of primers). Error bars represent \pm s.e.m.; $n=6$ technical replicates from two independent experiments $(*=$ $p<0.05$ by two-tailed student's $t$-tests). Shaded bars indicate known BRD4 binding sites.

Right panel: immunoblots, as indicated, of WCEs from transfected cells.

(d) RNA isolated from U2OS cells transfected with $3 \mu \mathrm{g}$ hBRD4, Mt BRD4 or control plasmid analyzed by qPCR for mRNA levels of $M Y C, A U R K B, F O S$ and AURKA. Error bars represent \pm s.e.m. (two-tailed student's $t$-tests); $n=4$ technical replicates from two independent experiments.

(e) ChIP of H3K122ac, H3K14ac and BRD4 around TSSs of MYC, AURKB and AURKA genes in U2OS cells transfected with $3 \mu \mathrm{g}$ hBRD4, Mt BRD4 or control plasmid that were treated with 500nM JQ1 or DMSO for 18hr prior to ChIP-qPCR. Error bars represent \pm 
s.e.m.; $n=4$ technical replicates from two independent experiments ( $p<0.05$ based on twotailed student's $t$-tests).

Uncropped images of immunoblots are shown in Supplementary Data Set 1 

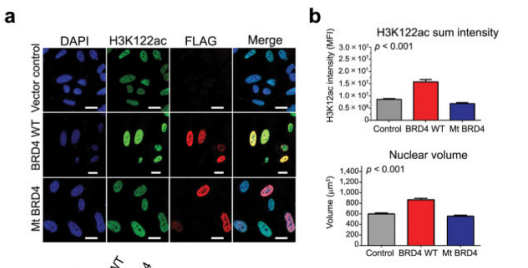

c
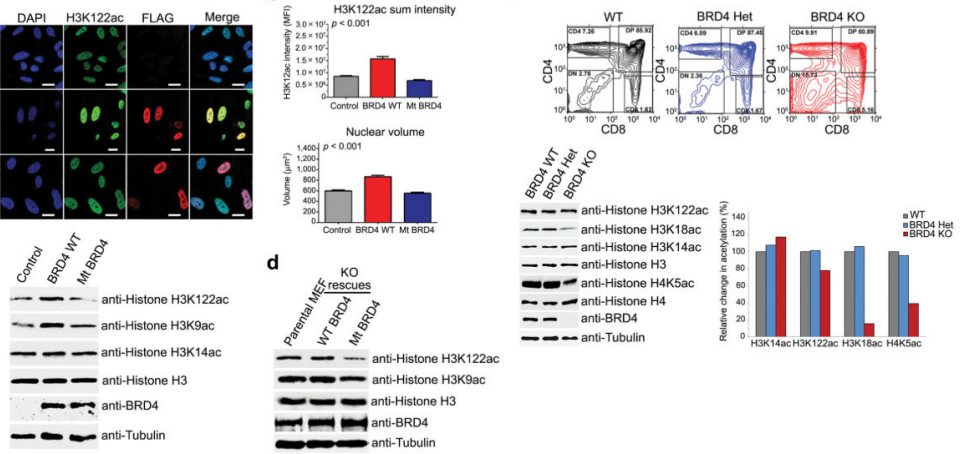

Fig. 7. BRD4 mediates chromatin de-compaction and thymic development

a) Representative IF and DAPI stained images of U2OS cells transfected with $3 \mu \mathrm{g}$ FlaghBRD4, Flag-MtBRD4 or control plasmid. Nuclear H3K122ac and Flag-BRD4 levels were visualized by immunofluorescence (IF) (upper panels). Scale bars, $20 \mu \mathrm{m}$. Immunoblots show H3K122ac, H3K9ac, AcHK14 and BRD4 levels in WCEs from transfected cells (lower panels).

(b) Relative levels of H3K122ac in U2OS cells transfected with hBRD4, MtBRD4 or control plasmid as quantified from IF analysis (upper panel). Quantitation of nuclear volume based on DAPI staining in U2OS cells (lower panel). Error bars represent s.e.m. $(n=100$ transfected cells) based on two-tailed Student's $t$-test.

(c) Flow cytometry profiles of thymocytes from wild type, heterozygous and BRD4 deficient mice stained for CD4 and CD8 (upper panels). Profile is representative of $>7$ thymi. Immunoblots of thymic WCE (bottom left panel). Changes in thymic H3K14ac, $\mathrm{H} 3 \mathrm{~K} 122 \mathrm{ac}, \mathrm{H} 3 \mathrm{~K} 18 \mathrm{ac}$ and $\mathrm{H} 4 \mathrm{~K} 5 \mathrm{ac}$ relative to total histone $\mathrm{H} 3$ and $\mathrm{H} 4$ quantified in graph (bottom right panel). Bars are average of two independent experiments with two mice. (d) Immunoblots of WCEs of parental BRD4 ${ }^{\mathrm{fl} / \mathrm{fl}} \mathrm{MEFs}$, BRD4-deleted MEFs (KO) rescued with either WT BRD4 or Mt BRD4, as indicated. (H3K122ac levels cannot be assessed in BRD4-deleted MEFs which do not proliferate.)

Uncropped images of immunoblots are shown in Supplementary Data Set 1

Nat Struct Mol Biol. Author manuscript; available in PMC 2016 November 09. 


\section{G1 gene bookmark \\ Histone acetylation \\ Nucleosome clearance \\ Chromatin de-compaction}
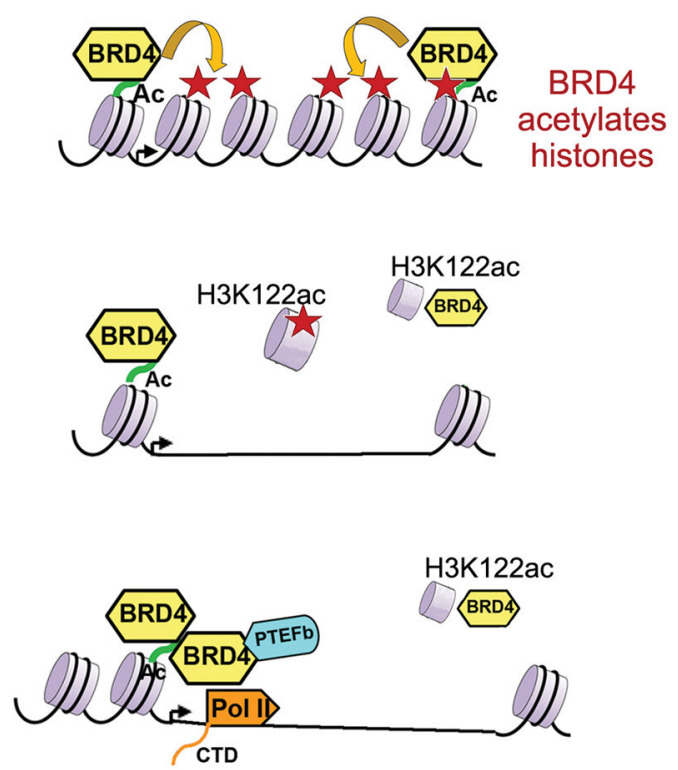

Fig. 8.

Model for the role of BRD4 histone acetyltransferase activity.

Cartoon shows BRD4 being recruited to acetylated histone lysines (e.g. H3K14) in nucleosomes associated with gene loci targeted for expression, such as the $M Y C$ and $F O S$ G1 genes. Once recruited to the chromatin, BRD4 acetylates histones, weakening histoneDNA interactions through the acetylation of tail lysines and globular H3K122, causing localized nucleosome disassembly and clearance, followed by increased transcription. 